\title{
Evaluation of safety and diagnostic yield of pleural cryobiopsies during thoracoscopy
} Mona M. Ahmed, Samar H. AlSharkawy, Amr M. Shoukri, Yousra T. AbouBakr

\author{
Background Biopsies using cryoprobe are now widely used \\ in interventional pulmonology. There are only few data \\ available on the use of cryotechnique through rigid \\ thoracoscopy.
}

Aim The aim of this work was to evaluate the diagnostic yield and safety of pleural cryobiopsies using rigid thoracoscopy.

Patients and methods A total of 30 patients with undiagnosed exudative pleural effusion who underwent rigid thoracoscopy were included in our study. Biopsies were obtained from suspicious areas by rigid forceps and cryoprobe.

Results A total of 30 patients with undiagnosed exudative pleural effusion have been analyzed. Histopathological results of thoracoscopic pleural biopsies revealed that the most common diagnosis was malignancy $(50 \%)$, followed by tuberculosis (26.7\%). Cryobiopsy specimens were larger than forceps biopsy specimens, with no difference in diagnostic yield $(76.7 \%)$. Cryotechnique proved to be beneficial in cases of thin highly vascular pleura, allowing easier biopsies with lesser risk of bleeding. In cases of

\section{Introduction}

Medical thoracoscopy is a useful, effective, and safe method for investigating and managing undiagnosed exudative pleural effusion [1].

Biopsy via cryoprobe, first used in 1968, has mainly been employed in the management of obstructive endobronchial tumors. Recently, cryotherapy via bronchoscopy is being used for several purposes including management of endobronchial tuberculosis, endobronchial tumors, diagnosis of interstitial lung disease, and investigating peripheral lung lesions. The cryoprobe is a feasible and efficient tool to obtain a large specimens with better preserved cellular architecture than specimens obtained through forceps biopsies, and also with better diagnostic yield [2,3].

Cryobiopsies of the pleura and lungs have been used with success during rigid thoracoscopy. There is also an analgesic effect of the cold, which made biopsies painless with preserved tissue architecture and minimal risk of hemorrhage [4].

\section{Patients and methods}

This study was conducted in the Department of Pulmonary Medicine at Ain Shams University Hospitals during a period of 1 year. It included 30 consecutive patients referred to our department thickened fibrous pleura, there was no difference between both the techniques.

Conclusion Pleural cryobiopsies through rigid thoracoscopy is a simple and safe procedure. It has a high diagnostic yield similar to rigid forceps biopsy, and it showed better performance in thin highly vascular pleura where biopsies can be performed with minimal risk of bleeding.

Egypt J Bronchol 2019 13:63-66

(C) 2019 Egyptian Journal of Bronchology

Egyptian Journal of Bronchology 2019 13:63-66

Keywords: cryobiopsies, forceps biopsies, thoracoscopy

Department of Chest Diseases, Faculty of Medicine, Ain Shams University, Cairo, Egypt

Correspondence to Amr M. Shoukri, MD, 43 ElMahrouky Street, Heliopolis, Cairo 11341, Egypt. Tel: +20 100660 1870;

e-mail: amr_shoukri@hotmail.com

Received 29 June 2018 Accepted 31 December 2018

with undiagnosed exudative pleural effusion, who underwent diagnostic rigid thoracoscopy. Written informed consent have been taken from all patients. The study was approved by the local institute ethics committee.

All patients are subjected to the following: full medical history including professional exposure to hazardous agents and history of any chronic disease; general examination and local chest examination; chest radiography and computed tomography chest with contrast; full laboratory investigations including bleeding profile, complete blood count, liver and kidney function tests, and other routine blood tests. Pleural fluid sampling was done for chemical (glucose, protein, lactate dehydrogenase, and adenosine deaminase), bacteriological (culture and sensitivity and Ziehl-Neelsen staining), and cytological examination. Fiberoptic bronchoscopy was performed in cases where parenchymal lung lesions were detected in computed tomography chest to investigate possible endobronchial lesions.

This is an open access journal, and articles are distributed under the term of the Creative Commons Attribution-NonCommercial-ShareAlike 4.0 License, which allows others to remix, tweak, and build upon the work non-commercially, as long as appropriate credit is given and the new creations are licensed under the identical terms. 


\section{Inclusion criteria}

Patients older than 18 years with undiagnosed exudative pleural effusion and no contraindications for thoracoscopy, and who sign the written informed consent to participate in the study were included.

\section{Exclusion criteria}

Patients with absolute or relative contraindications for thoracoscopy (coagulation disorders, thrombocytopenia, hemodynamic instability, and arrhythmias), any contraindication to general anesthesia, uncorrected hypoxemia despite oxygen administration, endobronchial lesions that may prevent lung re-expansion, and patients who refuse to participate in the study or refuse to sign the consent were excluded from the study.

\section{Thoracoscopy procedure}

In all patients, fasting for at least $6 \mathrm{~h}$ is required, with no other special preoperative preparation. The patients are monitored before and during the whole procedure (blood pressure, pulse, ECG, and pulse oximetry); the procedure was performed in lateral decubitus position with the affected side upward under general analgesia using a combination of inhalation anesthetic (isoflurane) and intravenous anesthetic (propofol).

Skin sterilization was performed followed by incision and blunt dissection in the appropriate intercostal space to enter the pleural space. A $10-\mathrm{mm}$ trocar was then inserted, and a $0^{\circ}$ telescope was inserted through it and connected to a video camera; the pleural space was carefully inspected through the thoracoscope (Tekno, Tuttlingen, Germany).

The appearance of the parietal and visceral pleural surfaces and the extent of their involvement were assessed visually through the thoracoscope.

Abnormal and suspicious areas of parietal pleura were biopsied (four to six biopsies by rigid forceps, and four to six biopsies by ERBE flexible cryoprobe).

Biopsy samples were sent in two different containers to the same pathologist who was blinded to the biopsy technique in each container.

Intraoperative comparison of average biopsy size, effectiveness on different pleural thickness, ability of nodules extraction, and biopsies-related complications was done. Following the procedure, a chest tube was inserted and after recovery chest radiograph was performed.
The cryosurgical equipment consists of the following: The console (ERBE ELEKTROMEDIZIN, Tübingen, Germany), with line voltage 230 volt, line frequency 50/ $60 \mathrm{~Hz}$, input current $0.2 \mathrm{~A}$, line fuse T0, $2 \mathrm{~A}$, type no 10443-000, and serial no C-1170.

Width $\times$ height $\times$ depth was $327 \times 960 \times 400 \mathrm{~mm}$.

Weight was $13.2 \mathrm{~kg}$

$\mathrm{CO}_{2}$ cylinder was used as cryogen.

Flexible cryoprobe, ERBE bronchoscopy cryoprobe, ref 20447-005, having diameter of $3 \mathrm{~mm}$ and length of $530 \mathrm{~mm}$ (ERBE, Germany) was used. The cryosurgical equipment operates by the Joule-Thompson effect, which dictates that a compressed gas released at high flow rapidly expands and creates a very low temperature, and therefore large pieces of tissue were extracted with the freeze-thaw cycle.

\section{Tissue sampling by cryoprobe}

The tip of the probe, cooled to $-89^{\circ} \mathrm{C}$ with $\mathrm{CO}_{2}$, was attached to the selected part of the parietal pleura for $30 \mathrm{~s}$. The frozen tissue was extracted by gently pulling. The probe with the attached biopsy sample was removed through the thoracoscope. Each biopsy sample was released from the probe by thawing in saline and was then fixed in formalin.

\section{Results}

A total of 30 patients with undiagnosed exudative pleural effusion were included in the present study. Their mean age was 54 years old, and $60 \%$ of patients were males.

All patients had exudative pleural effusion with different cytological results (Table 1). The most common thoracoscopic finding was pleural nodules in $30 \%$ of cases (Table 2).

The commonest diagnosis in our study was mesothelioma (46.7\%), and there was no difference in the diagnostic yield between forceps biopsy

Table 1 Pleural fluid cytology results among studied group

\begin{tabular}{lc}
\hline Pleural fluid cytology & $\begin{array}{c}N=30 \text { patients } \\
{[n(\%)]}\end{array}$ \\
\hline Exudative reaction with few mesothelial cells & $8(26.6)$ \\
Malignant effusion & $4(13.3)$ \\
Exudative reaction rich in lymphocytes & $15(50)$ \\
Hypocelluar exudate & $2(6.7)$ \\
Exudative reaction rich in eosinophils & $1(3.3)$ \\
\hline
\end{tabular}


Table 2 Thoracoscopic findings among studied group

\begin{tabular}{lc}
\hline Thoracoscopic finding & $N=30$ patients $[n(\%)]$ \\
\hline Thickened inflamed pleura & $8(26.7)$ \\
Pleural nodules & $9(30)$ \\
Pleural mass & $7(23.3)$ \\
Pleural and pulmonary nodules & $3(10)$ \\
Highly vascular pleura & $2(6.7)$ \\
Thickened fibrosed pleura with adhesions & $1(3.3)$ \\
\hline
\end{tabular}

Table 3 Rigid forceps biopsy histopathological results among studied group

\begin{tabular}{lc}
\hline Histopathological diagnosis & $N=30$ patients $[n(\%)]$ \\
\hline Mesothelioma & $14(46.7)$ \\
Tuberculosis & $8(26.7)$ \\
Nonspecific inflammation & $7(23.3)$ \\
Metastatic adenocarcinoma & $1(3.3)$ \\
Diagnostic yield (\%) & 76.7 \\
\hline
\end{tabular}

Table 4 Cryobiopsy histopathological results among studied group

\begin{tabular}{lc}
\hline Histopathological diagnosis & $N=30$ patients $[n(\%)]$ \\
\hline Mesothelioma & $14(46.7)$ \\
Tuberculosis & $8(26.7)$ \\
Nonspecific inflammation & $7(23.3)$ \\
Metastatic adenocarcinoma & $1(3.3)$ \\
Diagnostic yield $(\%)$ & 76.7 \\
\hline
\end{tabular}

specimens and cryobiopsy specimens (Tables 3 and 4). There were no recorded complications related to biopsy procedures using cryotechnique, whereas moderate bleeding, which was easily controllable, occurred when using forceps biopsies only in cases of thin highly vascular pleura.

In the studied group ( $n=30)$, we found that cryobiopsy was superior to rigid forceps biopsy in thin highly vascular pleura, which was found in only two $(6.7 \%)$ patients, larger biopsies and easier peeling was done using cryotechnique. There was no difference between both techniques regarding biopsy size in cases of thickened pleura. There was no difference in the technical difficulty between the two biopsy methods in cases of thickened nodular pleura.

\section{Discussion}

Thoracoscopy has been established as a valuable tool for the investigation of undiagnosed exudative pleural effusion, particularly for patients with high probability of malignancy [1].

Following assessment of the pleural cavity during thoracoscopy procedure, forceps biopsy is the commonest used instrument to obtain specimens from suspected pleural lesions; however, it may be associated with bleeding that hinders further biopsies. In additional, the decision to take a biopsy could be difficult, especially if the targeted lesions were on the visceral pleura or near a vascular structure [5].

A definitive diagnosis was reached in 23 out 30 cases, with diagnostic yield of $76.6 \%$. Fifteen (50\%) cases were diagnosed as having malignancy; 14 (46.7\%) cases had mesothelioma, and one (3.3\%) case metastatic adenocarcinoma. Tuberculosis was found in eight (26.7\%) cases. Nonspecific inflammation was found in seven $(23.3 \%)$ cases.

Malignancy was found to be the final diagnosis in most cases of undiagnosed pleural effusion as reported in several previous studies [6-8].

In the current study, the most common pathological type of malignancy was the malignant pleural mesothelioma, which was found in 14 (46.7\%) patients. This is probably owing to occupational exposure, and it is similar to other previous Egyptian studies [6,7]. Other studies may show different types of prevalence. Prabhu and Narasimhan [9] performed medical thoracoscopy for 68 patients, and 24 patients were diagnosed as having malignant pleural effusion. Unlike our study, the commonest type was metastatic adenocarcinoma, and mesothelioma was diagnosed in only three patients.

Our study demonstrated the feasibility and safety for obtaining biopsy specimens from parietal pleura using a cryoprobe during rigid thoracoscopy. There were no recorded adverse effects related to the procedure.

There was no difference in diagnostic yield between cryobiopsies and regular rigid forceps biopsies. Cryotechnique was found to be easier with lesser bleeding. This was particularly true for cases with thin and highly vascular pleura.

The difference between cryobiopsy and flexible forceps biopsy applied through semirigid thoracoscopy has been studied by in several studies [10-14].

Rozman et al. [10] found that cryobiopsy samples were bigger and significantly easier for interpretation than flexible forceps biopsy samples. Diagnostic yield was the same for both techniques, and there were no bleeding problems related to the procedure. In another study [12], the diagnostic yield was 99\% with cryobiopsy and 96\% with flexible forceps biopsy. The average specimen size through cryoprobe was significantly larger than with the conventional flexible forceps, and no major 
complications were noted. Maturu and Sehgal [14] reported that cryobiopsy was larger with better preserved cellular architecture and tissue integrity than flexible forceps biopsy. Forceps biopsy and cryobiopsy made definitive diagnoses in all cases.

Maturu and Sehgal [14] demonstrated that cryobiopsy was significantly superior to flexible forceps biopsy. The biopsies using the cryoprobe were larger, and also the diagnostic yield was higher.

All these previous studies used semirigid thoracoscopy to perform the procedure and flexible forceps to obtain biopsies, and this is different from our methodology, because we used rigid thoracoscope and rigid forceps for biopsy in comparison with cryobiopsy.

Wurps et al. [15] compared the two established biopsy techniques (rigid and flexible forceps biopsy) with the use of cryotechnique during medical thoracoscopy. In comparison, cryobiopsies showed a significantly larger biopsy size and depth than flexible forceps biopsies. On the contrary, rigid forceps biopsies showed significantly larger size and depth and higher diagnostic yield. Our results are not in accordance with this study, as we found that cryobiopsy was similar to rigid forceps biopsy in term of sample size in cases of thickened pleura, and superior to rigid forceps biopsy in cases with thin highly vascular pleura, and the diagnostic yield was similar for both techniques.

Rigid forceps biopsy is a well-established tool used through rigid thoracoscope, and cryotechnique has proved to be superior than flexible forceps using semirigid thoracoscope. The question raised in our study was the potential benefits that can be added when performing cryobiopsies through rigid thoracoscopy. Our conclusion is that cryobiopsy is beneficial in cases of thin highly vascular pleura, allowing easier biopsies with lesser risk of bleeding. In cases of thick fibrosed pleura, both techniques are similar in efficiency, and the diagnostic yield is also similar for both types of biopsies.

\section{Financial support and sponsorship} Nil.

\section{Conflicts of interest}

There are no conflicts of interest.

\section{References}

1 Rodriguez-Panadero F. Medical thoracoscopy. Respiration 2008; 76:363-372.

2 Fu EQ, Nan YD, Jin FG, Ma AQ. Therapeutic effects of sequential therapy by electric coagulation, cryotherapy and balloon dilation with an electronic video bronchoscope. Exp Ther Med 2013; 5:1649-1656.

3 Aktas Z, Gunay E, Hoca NT. Endobronchial cryobiopsy or forceps biopsy for lung cancer diagnosis. Ann Thorac Med 2010; 5:242-246.

4 Bonniot J, Homasson J, Roden S. Pleural and lung cryobiopsies during thoracoscopy. Chest 1989; 95:492-493.

5 Loddenkemper R, Grosser H, Gabler A, Mai J, Presseuler H, Brandt HJ. Prospective evaluation of biopsy methods in diagnosis of malignant pleural effusions: intra patient comparison between pleural fluid cytology, blind needle biopsy and thoracoscopy. Respir Dis 1983; 127:114.

6 Helala L, El-Assal G, Farghally A, Abd El Rady M. Diagnostic yield of medical thoracoscopy in cases of undiagnosed pleural effusion in Kobri El-Kobba Military Hospital. Egypt J Chest Dis Tuberc 2014; 63:629-634.

7 Abd El Rehim IY, Morsi AF, El-Shabrawy M, El Shahaat HA. The role of medical thoracoscopy in the diagnosis of exudative pleural effusion at the Chest Department of Zagazig University Hospitals. Egypt J Bronchol 2016; 10:225-231.

8 El daboosy M, El hamly M, Halima KM, Shaarawy AT, Alwakill OM. Medical video assisted thoracoscopy - minimally invasive diagnostic tool for diagnosis of undiagnosed pleural effusion. Egypt $J$ Chest Dis Tuberc 2013; 62:121-126.

9 Prabhu V, Narasimhan R. The role of pleuroscopy in undiagnosed exudative pleural effusion. Lung India 2012; 29:128-130.

10 Rozman A, Camlek L, Marc Malovrh M, Kern I, Schönfeld N. Feasibility and safety of parietal pleural cryobiopsy during semi-rigid thoracoscopy. Clin Respir J 2016; 10:574-578.

11 Vikas V, Shepherd RW, Hussein E, Malhotra R. Safety and feasibility of pleural cryobiopsy compared to forceps biopsy during semi-rigid pleuroscopy. Lung 2017; 195:371-375.

12 Tousheed SZ, Manjunath PH, Chandrasekar S, Murali Mohan BV, Kumar $\mathrm{H}$, Hibare KR, et al. Cryobiopsy of the pleura: an improved diagnostic tool. J Bronchology Interv Pulmonol 2018; 25:37-41.

13 Thomas R, Karunarathne S, Jennings B, Morey S, Chai SM, Lee YC, et al. Pleuroscopic cryoprobe biopsies of the pleura: a feasibility and safety study. Respirology 2015; 20:327-332.

14 Maturu VN, Sehgal IS. Pleuroscopic cryobiopsy: case series and systematic review. J Bronchol Interv Pulmonol 2015; 22:11-13.

15 Wurps H, Schönfeld N, Bauer T, Bock M, Duve C, Sauer R. Intrapatient comparison of parietal pleural biopsies by rigid forceps, flexible forceps and cryoprobe obtained during medical thoracoscopy: a prospective series of 80 cases with pleural effusion. BMC Pulm Med 2016; 16:98. 